\title{
Short telomere length is associated with arterial aging in patients with type 2 diabetes mellitus
}

\section{E N Dudinskaya, O N Tkacheva, M V Shestakova', N V Brailova, I D Strazhesko, D U Akasheva, 0 Y Isaykina, N V Sharashkina, D A Kashtanova and S A Boytsov}

National Research Center for Preventive Medicine, Building 10, Petroverigskiy Lane, Moscow 101990, Russian Federation

${ }^{1}$ Endocrinology Research Centre, Moscow, Russian Federation
Correspondence

should be addressed

to E N Dudinskaya

Email

katharina.gin@gmail.com

\begin{abstract}
It is known that glucose disturbances contribute to micro- and macrovascular complications and vascular aging. Telomere length is considered to be a cellular aging biomarker. It is important to determine the telomere length role in vascular structural and functional changes in patients with diabetes mellitus. We conducted a cross-sectional observational study in a high-risk population from Moscow, Russia. The study included 50 patients with diabetes and without clinical cardiovascular disease and 49 control group participants. Glucose metabolism assessment tests, measuring intima-media complex thickness and determining the presence of atherosclerotic plaques, pulse wave velocity measurement, and telomere length measurement were administered to all participants. Vascular changes were more dramatic in patients with diabetes than in the control group, and the telomeres were shorter in patients with diabetes. Significant differences were found in the vascular wall condition among diabetes patients, and there were no substantial differences in the arterial structure between patients with 'long' telomeres; however, there were statistically significant differences in the vascular wall condition between patients with 'short' telomeres. Vascular ageing signs were more prominent in patients with diabetes. However, despite diabetes, vascular changes in patients with long telomeres were very modest and were similar to the vascular walls in healthy individuals. Thus, long lymphocyte telomeres may have a protective effect on the vascular wall and may prevent vascular wall deterioration caused by glucose metabolism disorders.
\end{abstract}
Key Words
telomere length
- vascular ageing
- diabetes mellitus
- insulin resistance
- arterial stiffness

\section{Introduction}

Diabetes mellitus (DM) is a chronic noncommunicable disease that has reached epidemic proportions. Type $2 \mathrm{DM}$ (T2DM) inevitably leads to microvascular and macrovascular complications that aggravate the course and prognosis of cardiovascular diseases (CVD).

A vast body of evidence indicates that age-related endothelial dysfunction and the vascular wall thickening and stiffening set up a metabolically and enzymatically active environment that contributes to the manifestation and progression of CVD (1). Some processes that contribute to the vascular wall changes include: an increased collagen content accompanied by the formation of strong cross-links between fibers; elastin content fragmentation and reduction; accumulation of advanced glycation end products (AGE) (2); intima-media complex (IMT) thickening due to accumulation of extracellular matrix proteins http://www.endocrineconnections.org DOI: 10.1530/EC-15-0041
(C) 2015 The authors Published by Bioscientifica Ltd
This work is licensed under a Creative Commons Attribution-NonCommercial 4.0 International License. 
and smooth muscle cells; upregulation of adhesion molecules; and increased monocyte adhesion to the endothelial surface (3).

Experimental data show that glucose metabolism disorders induce vascular changes. For instance, Facchini et al. (4) showed that hyperinsulinaemia regardless of hyperglycaemia may promote oxidative stress and thereby accelerate vascular ageing and the development of agerelated diseases. In addition, insulin resistance (IR) is considered to be a predictor of atherosclerosis and CVD independently of other risk factors, such as blood lipid levels, and hyperglycaemia results in ageing, endothelial dysfunction, and arterial stiffness (5). Recently published studies demonstrate that arterial stiffness occurs in the initial stages of the glucose metabolism disorders, when IR is not yet accompanied by an increased glucose level $(6,7)$.

Differences in ageing rates in patients with T2DM may result from different 'genetic protection' levels. Some findings in vessel biology revealed the molecular mechanisms of ageing and methods of preventing or slowing down the arterial ageing process.

One of the most widely discussed genetic ageing markers is the peripheral blood lymphocyte telomere length. Lymphocyte telomeres are the ends of linear DNA that have the repetitive nucleotide sequence TTAGGG. Telomeres protect linear chromosome ends from degradation and fusion and maintain genome stability. Due to incomplete replication of the DNA ends in somatic cell, telomere is truncated in every mitosis cycle. As soon as telomere DNA becomes forbiddingly short, the cell loses the ability to maintain genomic integrity, repair DNA damages, maintain the metabolic activity, and divide $(8,9)$. Some authors describe the telomere as a 'molecular clock' that defines the cell's lifespan (10).

Experimental and clinical evidence indicates that the lymphocyte telomere length corresponds to the telomere length of the stem cells and endothelial progenitor cells. Thus, the lymphocyte telomere length could be used as a biomarker of vessel ageing. Therefore, the telomere length measuring in easily accessible tissues such as blood may be a substitute parameter for determining the telomere length in other tissues (11). The length and rate of telomere shortening are genetically determined; however, they are also influenced by external factors (12).

New data show an accelerated telomere shortening in patients with T2DM and impaired glucose tolerance $(13,14)$, and it could be related to the IR. Telomere length is considered to be a marker of T2DM and diabetes complications (15). Compared with healthy individuals, the presence of short telomeres has been demonstrated in patients with IR; however, even shorter telomeres were observed in patients with T2DM (16). Some data allow us to make a link between shortened lymphocyte telomeres and the development of T2DM, CVD and vascular ageing processes (17). These observations suggest that T2DM plays an important role in the processes of replicative senescence.

However, despite obvious scientific achievements in the field of vascular ageing, there are many unresolved issues, e.g. an insufficient number of ageing clinical studies in patients with T2DM; most published scientific studies had been mainly conducted on animals and cell cultures. Studying the relationship between the vascular structure and function changes and replicative senescence in patients with T2DM is of particular interest.

Studying the pathogenesis of changes in the cardiovascular system in patients with T2DM is highly relevant due to the steady ageing of the population, T2DM and CVD prevalence in the elderly, the lack of understanding of the ageing process in patients with diabetes, and the absence of effective methods to influence these processes.

\section{Aim}

The aim of this study is to investigate an association between the artery structural and functional changes and peripheral blood lymphocyte telomere length in T2DM.

\section{Materials and methods}

The cross-sectional observational study was conducted in a high-risk population from Moscow, Russia. Patients who previously underwent an outpatient examination in the FGBI National Research Centre for Preventive Medicine (NRCPM) during 2012-2013 were selected for this crosssectional study. The main study group included patients with T2DM with a disease duration of no longer than 12 months after diagnosis, HbA1c levels of 6.5-9.0\%, and 45-75 years old. The control group consisted of patients without T2DM and with no clinical CVD. The patients with diabetes and the controls were selected from a larger population-based study (Strazhesko ID, Tkacheva ON, Boytsov SA, Akasheva DU, Dudinskaya EN, Vygodin VA, Skvortsov DA, Nilsson PM, unpublished observations), which conducted similar analyses without focusing on the importance of type 2 diabetes in relation to the studied variables.

Criteria for exclusion from the study were as follows: T1DM and other specific types of diabetes; stage 3 arterial hypertension; blood pressure $>180 / 100 \mathrm{mmHg}$; regular use of anti-hypertensive drugs; regular use of anti-diabetic

This work is licensed under a Creative Commons Attribution-NonCommercial 4.0 International License. 
drugs; severe diabetic microangiopathy (pre-proliferative and proliferative diabetic retinopathy and stage $3 \mathrm{~b}, 4$, or 5 chronic kidney disease); clinical CVD with the New York Heart Association (NYHA) classification class II-IV chronic heart failure; presence of valvular heart disease; chronic liver and/or kidney failure; cancer; pregnancy; lactation; or refusal to participate in the study.

All of the patients signed a legal informed consent to participate in the study. The Local Ethics Committee (LEC) FGBI NRCPM Ministry of Healthcare, Russian Federation, approved this study protocol.

All of the patients underwent a standard clinical assessment during screening. The assessment included medical history; physical examination, height and weight measurements to calculate BMI, systolic (SBP) and diastolic blood pressure (DBP) measured using a calibrated instrument with shoulder cuff (HEM-7200 M3, Omron Healthcare, Kyoto, Japan). Blood pressure was measured on the right arm after a 10-min rest in the sitting position three times with 2-min intervals, and the average of the three measurements was used for analysis. Arterial hypertension was defined as blood pressure $\geq 140 / 90 \mathrm{mmHg}$. Blood samples were taken for clinical and biochemical laboratory tests. Rest and stress electrocardiograms (ECG) were recorded (treadmill test protocol BRUCE, Intertrack, Schiller, Miami, FL, USA). Patients with disorders, according to the data of blood tests, heart rhythm and/or cardiac conduction on ECG, and a positive stress test were excluded from the study.

Of the 158 patients screened, 99 were included in the study. Additional tests were conducted in all participants, including a glucose metabolism assessment; duplex scanning of the carotid arteries to measure IMT and determine the presence of atherosclerotic plaques; measurement of carotid-femoral pulse wave velocity (PWV); and telomere length measurement.

\section{Glucose metabolism assessment}

The glucose concentration was measured to assess the glucose metabolism using the glucose oxidase method on a SAPPHIRE-400 analyser using DiaSys diagnostic kits. The HbA1c level was measured by liquid chromatography on a SAPPHIRE400 (Niigata Mechatronics, Tokyo, Japan) analyser according to the manufacturer's standard procedure.

\section{Telomere length measurements}

The relative length of the peripheral blood lymphocyte DNA telomere was measured. The technique was based on a Cawthon study with some modifications (18).
The telomere DNA in the genome was estimated by realtime PCR. The genomic single-copy DNA was measured by parallel real-time PCR. It was assumed that the ratio of telomere and single-copy matrices was proportional to the lymphocyte telomeres length.

\section{Arterial stiffness measurements}

To evaluate the vascular wall condition, the carotidfemoral PWV was measured by applanation tonometry (SphygmoCor System, AtCor Medical, West Ryde, NSW, Australia). A high precision applanation tonometry was superimposed on the proximal (carotid) and distal (femoral) artery after a short time interval to record pulse waves. Central blood pressure, SBP, DBP, and ECG were recorded simultaneously with PWV. The distance travelled by the pulse wave between registration points was divided by the time needed, as determined by the time between the origin of pulsation and the R-wave position on the ECG to calculate PWV. A PWV value $>10 \mathrm{~m} / \mathrm{s}$ was considered to be an increased PWV.

\section{Evaluation of IMT and subclinical atherosclerosis}

The Q-LAB special application program (Philips, Eindhoven, The Netherlands) was used for duplex scanning of extracranial brachycephalic arteries in B-mode with parallel ECG recording. IMT was measured on the back wall of the common carotid artery (CCA). The sensor was located on the anterior and posterior margins of the $\mathrm{m}$. Sternoclaidomastoideus. Scanning was performed in three planes: two longitudinal planes and the transverse plane. CCA IMT was measured $1.5-2 \mathrm{~cm}$ proximal to bifurcation on the artery wall most remote from the sensor. Internal and external carotid arteries were evaluated at the point of the visual maximum thickening of diagnostic IMT scanning of the CCA. The structural evaluation of IMT included echogenicity analysis and assessment of the preserved layer structure. Echogenicity of the surrounding tissue was considered baseline when determining echogenicity of the intima. Echogenicity of the vessel lumen was used for the media. The standards proposed by the experts of the European Society of Hypertension and the European Society of Cardiology (2003) were used to assess CCA IMT. IMT $<0.9 \mathrm{~mm}$ was considered normal; increased thickness was $0.9-1.3 \mathrm{~mm}$ and diffuse IMT thickening $>1.3 \mathrm{~mm}$ was considered as atherosclerosis. The presence of atherosclerotic plaques was defined as an increase in IMT $>1.3 \mathrm{~mm}$ in CCA, a local increase in IMT of $0.5 \mathrm{~mm}$ or a $50 \%$ increase in nearby IMT. Local IMT thickening

This work is licensed under a Creative Commons Attribution-NonCommercial 4.0 International License. 
$>1.3 \mathrm{~mm}$, which caused stenosis of the lumen but did not affect its internal anatomy, was considered as a plaque.

\section{Statistical analysis}

SAS 9.1 Statistical Software (SAS Institute, Cary, NC, USA) was used for analysis. All data were entered into a spreadsheet, and exploratory analysis was performed to identify data entry errors and missing values. Tests of skewness and kurtosis were performed for quantitative parameters and revealed a normal distribution for the majority of the quantitative parameters. Quantitative data are presented as mean and mean-square deviation. Comparative analysis of independent samples was conducted. Mean values of the clinical parameters were compared between the two groups using one-way ANOVA for continuous variables and the $\chi^{2}$ test for categorical variables. A modified Student's $t$-test was used with Fisher's arcsine transformation for the frequencies of the qualitative parameters. Pearson's linear correlation analysis was used to detect correlations between parameters. Multivariate regression equations and multiple linear regression analyses were used to identify which correlations between parameters were independent. The null hypothesis was rejected at $P<0.05$.

\section{Results}

In total, 99 patients (33 males and 66 females) were included in the study. The mean age was $52.4 \pm 12.3$ years. The patients were divided into two groups: those with T2DM $(n=50)$ and those without T2DM $(n=49)$. The patient groups were comparable in age and sex. No significant differences in the number of males/females were observed between the groups. T2DM duration was $0.9 \pm 0.089$ years. BMI in the T2DM group was significantly higher than that in the group of healthy individuals. Blood pressure did not differ significantly between the groups. Telomere length was significantly shorter in the T2DM group than in the control group. Fasting blood glucose (FBG) and HbA1c levels in patients with T2DM were significantly higher than those in the control group.

A significantly higher PWV and thickened IMT were observed in patients with T2DM than in the control group. The number of atherosclerotic plaques tended to be higher in the T2DM group than in the control group $(P=0.08)$. The main patient characteristics are shown in Table 1.

All of the patients were divided into two groups according to the relative peripheral telomere length. The median telomere length was 9.75. All the patients with a telomere length less than the median were assigned to the 'short' telomere group, and patients with a telomere length equal to or above the median were assigned to the 'long' telomere group.

The vascular wall status and glucose metabolism parameters were compared with the telomere length in all the patients.

The severity of subclinical atherosclerosis and vascular stiffness were higher in patients with short telomeres than in those with long telomeres in both groups. In contrast, in patients with T2DM and long telomeres, indicators of vascular ageing were significantly less frequent than in those with T2DM and short telomeres: PWV and IMT in the long telomere group were significantly lower $(P<0.01$ for both parameters) and the number of atherosclerotic plaques was significantly fewer $(P=0.03)$ (Table 2$)$.

The HbA1c level in patients with T2DM and short telomeres was significantly higher than in patients with T2DM and long telomeres. Patients with T2DM and short telomeres had higher rates of PWV $(P<0.01)$ and IMT $(P=0.03)$ and a greater number of atherosclerotic plaques $(P=0.04)$ than subjects without diabetes and with short telomeres. The vascular stiffness and subclinical atherosclerosis indicators did not differ in the T2DM group with long telomeres and the control group: PWV and IMT were comparable in participants with and without T2DM ( $P=0.91$ and $P=0.12$, respectively) and the number of atherosclerotic plaques did not differ significantly $(P=0.97)$ (Table 2$)$.

Table 1 Main clinical characteristics, results of duplex scanning of the carotid arteries and applanation tonometry and telomere length.

\begin{tabular}{|c|c|c|c|}
\hline Characteristic & $\begin{array}{c}\text { T2DM+ } \\
(n=50)\end{array}$ & $\begin{array}{l}\text { T2DM - } \\
(n=49)\end{array}$ & $\boldsymbol{P}$ \\
\hline Age (years) & $56 \pm 12.1$ & $53.47 \pm 11.91$ & 0.15 \\
\hline Male $(n / \%)$ & $15 / 30$ & $17 / 34$ & 0.77 \\
\hline BMI $\left(\mathrm{kg} / \mathrm{m}^{2}\right)$ & $31.1 \pm 1.08$ & $26.6 \pm 0.53$ & 0.002 \\
\hline $\mathrm{SBP}(\mathrm{mmHg})$ & $129.6 \pm 3.2$ & $123.3 \pm 1.5$ & 0.06 \\
\hline $\mathrm{DBP}(\mathrm{mmHg})$ & $79.06 \pm 1.8$ & $77.2 \pm 0.9$ & 0.37 \\
\hline T2DM duration (years) & $0.9 \pm 0.089$ & & \\
\hline HbA1c (\%) & $7.2 \pm 0.6$ & $5.09 \pm 0.05$ & $<0.001$ \\
\hline FBG $(\mathrm{mmol} / \mathrm{l})$ & $8.1 \pm 0.333$ & $5.3 \pm 0.051$ & $<0.001$ \\
\hline PWV (m/s) & $13.07 \pm 0.6$ & $10.67 \pm 0.23$ & $<0.001$ \\
\hline IMT (mm) & $0.88 \pm 0.02$ & $0.74 \pm 0.01$ & $<0.001$ \\
\hline $\begin{array}{c}\text { Number of athero- } \\
\text { sclerotic plaques }\end{array}$ & $1.3 \pm 0.2$ & $0.84 \pm 0.1$ & 0.08 \\
\hline $\begin{array}{l}\text { Relative telomere } \\
\text { length }\end{array}$ & $9.53 \pm 0.1$ & $9.86 \pm 0.1$ & 0.02 \\
\hline
\end{tabular}

SBP, systolic blood pressure; DBP, diastolic blood pressure; FBG, fasting blood glucose; PWV, carotid-femoral pulse wave velocity; IMT, intimamedia thickness. 
Table 2 Parameters for vascular wall status depending on the presence of T2DM and telomere length.

\begin{tabular}{|c|c|c|c|c|c|}
\hline \multirow[b]{2}{*}{ Parameter } & \multicolumn{2}{|c|}{ Long telomeres } & \multicolumn{2}{|c|}{ Short telomeres } & \multirow[b]{2}{*}{$P$} \\
\hline & $\mathrm{T} 2 \mathrm{DM}+(n=29)$ & $\mathrm{T} 2 \mathrm{DM}-(n=27)$ & $\mathrm{T} 2 \mathrm{DM}+(n=21)$ & $\mathrm{T} 2 \mathrm{DM}-(n=22)$ & \\
\hline PWV (m/s) & $10.50 \pm 0.1$ & $10.51 \pm 0.51$ & $15.08 \pm 1.31$ & $10.7 \pm 0.52$ & $\begin{array}{c}P^{1}=0.91 \\
P^{2}<0.01 \\
P^{3}<0.01 \\
P^{4}=0.025\end{array}$ \\
\hline IMT (mm) & $0.80 \pm 0.09$ & $0.73 \pm 0.03$ & $0.87 \pm 0.05$ & $0.78 \pm 0.13$ & $\begin{array}{l}P^{1}=0.12 \\
P^{2}=0.03 \\
P^{3}<0.01 \\
P^{4}=0.04\end{array}$ \\
\hline $\begin{array}{l}\text { Number of athero- } \\
\text { sclerotic plaques }\end{array}$ & $0.76 \pm 0.04$ & $0.78 \pm 0.02$ & $1.02 \pm 0.29$ & $0.89 \pm 0.22$ & $\begin{array}{l}P^{1}=0.97 \\
P^{2}=0.04 \\
P^{3}=0.03 \\
P^{4}=0.03\end{array}$ \\
\hline
\end{tabular}

$P^{1}$, vascular wall characteristics differences in diabetic and nondiabetic patients with long telomeres; $P^{2}$, vascular wall characteristics differences in diabetic and nondiabetic patients with short telomeres; $P^{3}$, vascular wall characteristics differences in diabetic patients with long and short telomeres; $P^{4}$, vascular wall characteristics differences in nondiabetic patients with long and short telomeres.

Table 3 shows correlation analysis results for PWV and IMT with other parameters in participants with and without T2DM. PWV was significantly correlated with SBP, IMT, and HbA1c. A significant negative correlation was detected between PWV and the telomere length in the T2DM group. IMT was significantly correlated with SBP and negatively with the telomere length. Also such correlation was observed between PWV and age, SBP and IMT, while a negative correlation was detected between PWV and telomere length in the control group. IMT was significantly correlated with age and SBP.

Analysis of the telomere length in the T2DM group demonstrated an inverse correlation between the telomere length, HbA1c, and PWV (Table 4).
Multiple linear regression analysis with the telomere length as the dependent variable and age, PWV, FBG, and HbA1c as independent variables showed that only PWV (inverse relationship) and HbA1c (direct relationship) were correlated with the telomere length (Table 5).

\section{Discussion}

We revealed that the vascular wall condition in patients with T2DM was significantly different from that in healthy individuals. Our results are consistent with those of other studies and have a pathophysiological basis (19). One of the possible explanations for increased vascular wall rigidity in patients with T2DM is an AGE accumulation

Table 3 Pearson's correlation analysis between pulse wave velocity (PWV) and intima-media thickness (IMT) and other parameters.

\begin{tabular}{|c|c|c|}
\hline \multirow[b]{2}{*}{ Parameter } & \multicolumn{2}{|c|}{$\mathbf{T 2 D M}+(n=50)$} \\
\hline & $\mathrm{PWV}(\mathrm{m} / \mathrm{s})$ & IMT (mm) \\
\hline Age (years) & $\begin{array}{c}0.1953 \\
P=0.17\end{array}$ & $\begin{array}{c}0.3564 \\
P=0.1501\end{array}$ \\
\hline $\mathrm{SBP}(\mathrm{mmHg})$ & $\begin{array}{c}0.2717 \\
P=0.003\end{array}$ & $\begin{array}{c}0.3231 \\
P=0.007\end{array}$ \\
\hline $\mathrm{DBP}(\mathrm{mmHg})$ & $\begin{array}{c}0.0983 \\
P=0.27502\end{array}$ & $\begin{array}{c}0.2196 \\
P=0.133\end{array}$ \\
\hline BMI $\left(\mathrm{kg} / \mathrm{m}^{2}\right)$ & $\begin{array}{c}0.3127 \\
P=0.001\end{array}$ & $\begin{array}{c}0.1731 \\
P=0.142\end{array}$ \\
\hline $\mathrm{FBG}(\mathrm{mmol} / \mathrm{l})$ & $\begin{array}{c}0.3621 \\
P=0.301\end{array}$ & $\begin{array}{c}0.2258 \\
P=0.0674\end{array}$ \\
\hline HbA1c (\%) & $\begin{array}{c}0.3526 \\
P=0.002\end{array}$ & $\begin{array}{c}0.1571 \\
P=0.0699\end{array}$ \\
\hline Relative telomere length & $\begin{array}{l}-0.3564 \\
P=0.019\end{array}$ & $\begin{array}{c}-0.3184 \\
P=0.0278\end{array}$ \\
\hline
\end{tabular}

\begin{tabular}{|c|c|}
\hline \multicolumn{2}{|c|}{ T2DM - $(n=49)$} \\
\hline PWV (m/s) & IMT (mm) \\
\hline 0.3213 & 0.3644 \\
\hline$P=0.001$ & $P=0.0001$ \\
\hline 0.3784 & 0.3214 \\
\hline$P=0.0021$ & $P=0.0214$ \\
\hline 0.01024 & 0.0538 \\
\hline$P=0.2765$ & $P=0.4245$ \\
\hline 0.0054 & 0.02985 \\
\hline$P=0.8594$ & $P=0.4211$ \\
\hline 0.1738 & 0.1732 \\
\hline$P=0.1422$ & $P=0.1421$ \\
\hline 0.1528 & 0.1635 \\
\hline$P=0.152$ & $P=0.0672$ \\
\hline-0.3623 & 0.1673 \\
\hline$P=0.0014$ & $P=0.0711$ \\
\hline
\end{tabular}

SBP, systolic blood pressure; DBP, diastolic blood pressure; FBG, fasting blood glucose.

$\begin{array}{lr}\text { http://www.endocrineconnections.org } & \text { ( } 2015 \text { The authors } \\ \text { DOI: } 10.1530 / \text { EC-15-0041 } & \text { Published by Bioscientifica Ltd }\end{array}$


Table 4 Correlation analysis of relative telomere length and other parameters in patients with T2DM.

\begin{tabular}{|c|c|c|}
\hline \multirow[b]{2}{*}{ Parameter } & \multicolumn{2}{|c|}{ Telomere length } \\
\hline & $r$ & $P$ \\
\hline Age (years) & 0.025 & 0.87 \\
\hline SBP $(\mathrm{mmHg})$ & -0.03 & 0.84 \\
\hline $\mathrm{DBP}(\mathrm{mmHg})$ & 0.12 & 0.5 \\
\hline BMI $\left(\mathrm{kg} / \mathrm{m}^{2}\right)$ & -0.02 & 0.85 \\
\hline FBG (mmol/l) & -0.31 & 0.52 \\
\hline HbA1c (\%) & -0.31 & 0.03 \\
\hline PWV (m/s) & -0.35 & $<0.01$ \\
\hline IMT (mm) & -0.11 & 0.41 \\
\hline $\begin{array}{l}\text { Number of atherosclerotic } \\
\text { plaques }\end{array}$ & -0.13 & 0.14 \\
\hline
\end{tabular}

(20) that leads to the formation of cross-links between collagen molecules in the middle layer of the vascular wall, resulting in increased collagen rigidity and vascular wall stiffness. The presence of chronic hyperglycaemia in patients with T2DM amplifies protein glycation and AGE accumulation and results in significantly increased vascular stiffness and therefore in accelerated vascular wall ageing $(19,20)$. We demonstrated a correlation between arterial stiffness and $\mathrm{HbA} 1 \mathrm{c}$, which is the main indicator of glucose metabolism.

Our results demonstrated that the peripheral lymphocyte telomere length was shorter in patients with T2DM than in healthy individuals. A similar correlation was demonstrated in the study conducted by Hovatta et al. (16). However, Sampson et al. (21) found no correlation between lymphocyte telomere shortening and glucose metabolism indicators in a European study, possibly because of the small number of patients in that study. Besides the significant differences in the HbA1c level between T2DM patients with long telomeres and this with short telomeres, this study also shows a negative correlation between the telomere length and HbA1c. These data suggest the damaging effect of hyperglycaemia on replicative ageing indicators. However, this finding needs additional larger scale research.

The most important results of this study are the finding of the independent negative relationship between the telomere length and PWV and independent positive association between the telomere length and HbA1c in T2DM patients. In other words, shorter telomeres in patients with T2DM are associated with stiffer vessels and poor diabetes control. The main cause of lymphocyte telomere shortening during the lifetime is an oxidative stress and the patterns associated with oxidative stress (obesity, IR, and chronic stress). Inflammation is associated with enhanced cell proliferation that leads to rapid telomere length shortening, and oxidative stress causes the single-stranded DNA breaks in telomere repeats promoting the accelerated telomeres' shortening during the repeated divisions.

In DM patients the telomere length shortening is more prominent due to damaging effects of chronic hyperglycemia, AGE accumulation, etc. One of the AGE agents is HbA1c. It is known that the level of HbA1c determines the vascular changes rate and that $\mathrm{HbA1c}$ is known to be predictive of future CVD independent of FBG. Some clinical studies have shown an association between the rates of the telomere shortening and the presence of T2DM $(6,22)$. It is also known that IR in T2DM is accompanied by endothelial dysfunction. The deficit in NO is considered to be the link between these processes. In cultured cells it has also been shown that IR may induce the smooth muscle cell proliferation and migration leading to vascular stiffness (3). Perhaps it is the telomere shortening that, in association of $\mathrm{T} 2 \mathrm{DM}$, results in the vascular aging and the T2DM-related CVD development, although this hypothesis requires further investigation and clarification.

Another important finding of this study is the evidence that the vascular walls in patients with T2DM and long lymphocyte telomeres were not significantly different from vascular walls in healthy individuals without T2DM. This finding indicates that genetically long telomeres may prevent accelerated vessel ageing in patients with type 2 diabetes of short duration (patients with established diabetes duration $<1$ year were included). Also, as all of the patients with diabetes were free of drug treatment, the results were not influenced by any drugs.

In contrast, patients with T2DM and short lymphocyte telomeres demonstrated a higher vascular stiffness and subclinical atherosclerosis severity despite the relatively short diabetes duration. Notably, participants with and without T2DM were comparable in age and SPB/DBP readings and ratio. Therefore, the influence of age and blood pressure on the telomere length was comparable.

Table 5 Multiple linear regression analysis of telomere length based on age, FBG, HbA1C and PWV as independent variables in the T2DM.

\begin{tabular}{|c|c|c|c|}
\hline Parameter & $\beta$ & S.E.M. & $P$ \\
\hline Age (years) & 0.029 & 0.530 & 0.85 \\
\hline PWV (m/s) & -0.15 & 2.721 & 0.037 \\
\hline $\mathrm{FBG}(\mathrm{mmol} / \mathrm{l})$ & -0.02 & 0.537 & 0.98 \\
\hline HbA1c (\%) & 0.067 & 0.841 & 0.036 \\
\hline
\end{tabular}

(7) This work is licensed under a Creative Commons Attribution-NonCommercial 4.0 International License. 
Thus, short telomeres were associated with rigid blood vessels, and long telomeres were associated with a betterpreserved vascular wall.

One possible explanation is that lymphocytes are used to determine the telomere length in clinical practice, which essentially reflects the telomere length in stem and progenitor cells. These cells are involved in damaged tissue repairing processes and tissue differentiation and play an important role in maintaining the tissue homeostasis and ensuring the preservation of endothelial function. However, blood vessel stiffness is mainly determined by the condition of the extracellular matrix; thus, cells may be present in the matrix, and the replicative activity of these cells affects vascular stiffness. Slow telomere shortening is likely to affect the matrix by some other mechanism, but not by the replicative activity.

Increasing evidence suggests that lymphocyte telomere shortening is a key component diminishing stem cell facilities and age-related tissue degeneration including vascular rigidity (20). However, the explanation is still lacking.

\section{Conclusion}

In summary, our observational cross-sectional study in a high CVD risk population showed the correlations between T2DM, cellular ageing processes, and the severity of subclinical morpho-functional changes in the vascular wall explained the higher incidence of CVD in patients with T2DM. Prevention of such changes may help avert the occurrence of CVD in patients with T2DM, particularly in patients with short peripheral lymphocyte telomeres.

\section{Declaration of interest}

The authors declare that there is no conflict of interest that could be perceived as prejudicing the impartiality of the research reported.

\section{Funding}

This research did not receive any specific grant from any funding agency in the public, commercial or not-for-profit sector.

\begin{abstract}
Author contribution statement
E N Dudinskaya, I D Strazhesko and D U Akasheva: development of the conception and design, analysis and data interpretation. O N Tkacheva: development of the conception and design, analysis and data interpretation, final statement for publication of the manuscript. M V Shestakova and S A Boytsov: final statement for publication of the manuscript. $\mathrm{N} V$ Brailova and D A Kashtanova: analysis and data interpretation. O Y Isaykina: PWV measurement. N V Sharashkina: IMT measuring.
\end{abstract}

\section{Acknowledgements}

The authors would like to thank A S Kruglikova, E V Plokhova, V S Pykhtina from the FGBI National Research Centre for Preventive Medicine, Moscow Russian Federation, and D A Skvortsov from the Lomonosov Moscow State University for research assistance.

\section{References}

1 Nilsson PM, Boutouyrie P, Cunha P, Kotsis V, Narkiewicz K, Parati G, Rietzschel E, Scuteri A \& Laurent S. Early vascular ageing in translation: from laboratory investigations to clinical applications in cardiovascular prevention. Journal of Hypertension 201331 1517-1526. (doi:10.1097/ HJH.0b013e328361e4bd)

2 Benetos A, Okuda K, Lajemi M, Kimura M, Thomas F, Skurnick J, Labat C, Bean K \& Aviv A. Telomere length as an indicator of biological aging: the gender effect and relation with pulse pressure and pulse wave velocity. Hypertension 200337 381-385. (doi:10.1161/01.HYP.37.2.381)

3 Scuteri A, Najjar SS, Muller DC, Andres R, Hougaku H, Metter EJ \& Lakatta EG. Metabolic syndrome amplifies the age-associated increases in vascular thickness and stiffness. Journal of the American College of Cardiology 200443 1388-1395. (doi:10.1016/j.jacc.2003.10.061)

4 Facchini FS, Hua N, Abbasi F \& Reaven GM. Insulin resistance as a predictor of age-related diseases. Journal of Clinical Endocrinology and Metabolism 200186 3574-3578. (doi:10.1210/jcem.86.8.7763)

5 Kozakova M, Morizzo C, Bianchi C, Di Filippi M, Miccoli R, Paterni M, Di Bello V \& Palombo C. Glucose-related arterial stiffness and carotid artery remodeling: a study in normal subjects and type 2 diabetes patients. Journal of Clinical Endocrinology and Metabolism 201499 E2362-E2366. (doi:10.1210/jc.2014-2028)

6 Gardner JP, Li S, Srinivasan SR, Chen W, Kimura M, Lu X, Berenson GS $\&$ Aviv A. Rise in insulin resistance is associated with escalated telomere attrition. Circulation 2005111 2171-2177. (doi:10.1161/01.CIR. $0000163550.70487 .0 B)$

7 Chen S, Yeh F, Lin J, Matsuguchi T, Blackburn E, Lee E, Howard BV \& Zhao J. Short leukocyte telomere length is associated with obesity in American Indians: the strong heart family study. Aging 20146 380-389.

8 Samani NJ, Boultby R, Butler R, Thompson JR \& Goodall AH. Telomere shortening in atherosclerosis. Lancet 2001358 472-473. (doi:10.1016/ S0140-6736(01)05633-1)

9 Benetos A, Gardner JP, Zureik M, Labat C, Xiaobin L, Adamopoulos C, Temmar M, Bean KE, Thomas F \& Aviv A. Short telomeres are associated with increased carotid atherosclerosis in hypertensive subjects. Hypertension 200343 182-185. (doi:10.1161/01.HYP.0000113081. 42868.f4)

10 Blackburn EH, Greider CW \& Szostak JW. Telomeres and telomerase: the path from maize, Tetrahymena and yeast to human cancer and aging. Nature Medicine 200612 1133-1138. (doi:10.1038/nm10061133)

11 Daniali L, Benetos A, Susser E, Kark JD, Labat C, Kimura M, Desai K, Granick M \& Aviv A. Telomeres shorten at equivalent rates in somatic tissues of adults. Nature Communications 20134 1597. (doi:10.1038/ ncomms2602)

12 Aviv A. Telomeres and human aging: facts and fibs. Science of Aging Knowledge Environment 200451 pe43. (doi:10.1126/sageke.2004.51. pe43)

13 Murillo-Ortiz B, Albarrán-Tamayo F, Arenas-Aranda D, BenítezBribiesca L, Malacara-Hernández JM, Martínez-Garza S, HernándezGonzález M, Solorio S, Garay-Sevilla ME \& Mora-Villalpando C. Telomere length and type 2 diabetes in males: a premature aging syndrome. Aging Male 201215 54-58. (doi:10.3109/13685538.2011. 593658)

14 Mulder $\mathrm{H}$. Is shortening of telomeres the missing link between aging and the type 2 diabetes epidemic? Aging 20102 634-636.

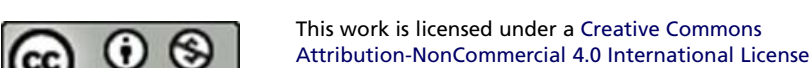


15 Jeanclos E, Krolewski A, Skurnick J, Kimura M, Aviv H, Warram JH \& Aviv A. Shortened telomere length in white blood cells of patients with IDDM. Diabetes 199847 482-486. (doi:10.2337/diabetes.47.3.482)

16 Hovatta I, de Mello VD, Kananen L, Lindström J, Eriksson JG, Ilanne-Parikka P, Keinänen-Kiukaanniemi S, Peltonen M, Tuomilehto J \& Uusitupa M. Leukocyte telomere length in the Finnish Diabetes Prevention Study. PLoS ONE 20127 e34948. (doi:10.1371/journal. pone.0034948)

17 Shah AS, Dolan LM, Kimball TR, Gao Z, Khoury PR, Daniels SR \& Urbina EM. Influence of duration of diabetes, glycemic control, and traditional cardiovascular risk factors on early atherosclerotic vascular changes in adolescents and young adults with T2DM. Journal of Clinical Endocrinology and Metabolism 200994 3740-3745. (doi:10.1210/jc.2008-2039)

18 Cawthon RM. Telomere measurement by quantitative PCR. Nucleic Acids Research 200230 e47. (doi:10.1093/nar/30.10.e47)
19 Sell DR \& Monnier VM. Molecular basis of arterial stiffening: role of glycation - a mini-review. Gerontology 201258 227-237. (doi:10.1159/ 000334668)

20 Sharpless NE \& DePinho RA. How stem cells age and why this makes us grow old. Nature Reviews. Molecular Cell Biology 20078 703-713. (doi:10.1038/nrm2241)

21 Sampson MJ, Winterbone MS, Hughes JC, Dozio N \& Hughes DA. Monocyte telomere shortening and oxidative DNA damage in type 2 diabetes. Diabetes Care 200629 283-289. (doi:10.2337/diacare.29.02. 06.dc05-1715)

22 Kilhovd BK, Juutilainen A, Lehto S, Rönnemaa T, Torjesen PA, Hanssen KF \& Laakso M. Increased serum levels of advanced glycation endproducts predict total, cardiovascular and coronary mortality in women with T2DM: a population-based 18 year follow-up study. Diabetologia 200750 1409-1417. (doi:10.1007/s00125-007-0687-z)

Received in final form 26 May 2015

Accepted 28 May 2015 http://www.endocrineconnections.org DOI: 10.1530/EC-15-0041
(C) 2015 The authors Published by Bioscientifica Ltd
This work is licensed under a Creative Commons Attribution-NonCommercial 4.0 International License. 